\title{
Pharmacologically Induced Hypertension Therapy for Acute Stroke Patients
}

\author{
Min Hwan Lee, Joong-Goo Kim, Sang Bum Jeon, Dong-Wha Kang, Sun U. Kwon, Jong S. Kim \\ Department of Neurology, Asan Medical Center, University of Ulsan College of Medicine, Seoul, Korea
}

\section{Dear Sir:}

In patients who experience acute stroke, excessive blood pressure (BP) lowering may result in early neurological deterioration (END) ${ }^{1}$ and unfavorable clinical outcomes. ${ }^{2}$ Thus, pharmacologically induced hypertension (PIH) may be effective for patients with signs of END. Although a few studies have reported potential benefits, ${ }^{3-7}$ the feasibility and safety of PIH remain unclear. We aimed to identify the characteristics of patients who require $\mathrm{PIH}$ therapy and to examine the feasibility, safety, and potential efficacy of PIH.

At the Asan Medical Center, we have been performing PIH therapy since 2011 for patients who exhibit END or are at increased risk for END, such as those with fluctuating neurological symptoms, based on the results from previous studies. ${ }^{4,5,7} \mathrm{Al}-$ though END was defined as an increase in National Institutes of Health Stroke Scale (NIHSS) score $\geq 2$, patients with a 1-point increase were occasionally included according to the attending neurologist's judgment. PIH was not performed in patients with systolic BP $>180 \mathrm{~mm} \mathrm{Hg}$ or diastolic BP $>110 \mathrm{~mm} \mathrm{Hg}$, ventricular tachycardia, or symptomatic heart or renal failure. However, because PIH is not an established treatment, some patients with END were treated by augmenting fluid therapy without PIH according to the discretion of attending physicians. For PIH, BP elevation of $15 \%$ to $25 \%$ over baseline was targeted, with a maximum systolic BP of $220 \mathrm{~mm} \mathrm{Hg}{ }^{3,5}$ Phenylephrine $(50 \mathrm{mg}$ ) mixed with $250 \mathrm{~mL}$ of normal saline was intravenously administered at a rate of $0.3 \mu \mathrm{g} / \mathrm{kg} / \mathrm{min}$ and titrated every 30 to 60 minutes. PIH was usually maintained for 5 days and tapered thereafter. If END recurred, PIH was re-administered for several days.

Two groups were selected as controls: the first was matched with age and sex (CT1) and, the second, with an additional stroke subtype (CT2). Considering the expected unfavorable out- come in patients with $E N D,{ }^{8}$ a favorable functional outcome was defined as a modified Rankin Scale (mRS) score of $\leq 3$ at 3 months post-stroke. "Early responsiveness" was defined when patients exhibited neurological improvement (NIHSS score $\geq 2$ improvement or physician's documentation for neurological improvement) within 1 day after PIH initiation. BP was recorded at four time points: the first-measured hospital BP (initial BP [iBP]); $\mathrm{BP}$ checked immediately before starting PIH (baseline BP [BPO]); BP checked at 7:00 AM 1 day after starting PIH (BP1); and BP checked at 7:00 AM 1 day after completing PIH (end BP [eBP]). This study was approved by the Institutional Review Board of Asan Medical Center, and the requirements for informed written consent was waived.

Between 2011 and 2016, 121 patients were treated with PIH (mean \pm standard deviation age, $65.9 \pm 12.7$ years; women, 40\%). Compared with CT1 patients, PIH patients more often had small vessel disease (SVD, $42.3 \%$ vs. 19.8\%) and large artery disease (LAD, $41.3 \%$ vs. $31.4 \%)$, and less often experienced cardioembolic stroke ( $4.15 \%$ vs. $34.7 \%)(P<0.001$, chi-square test). As stroke subtype may be associated with patient outcome, subsequent analyses was performed with CT2 patients. PIH patients had significantly higher systolic iBP, higher initial NIHSS score, and more often had END compared with CT2 patients (Table 1).

In the PIH group, the mean arterial pressure of BPO $(96.9 \pm 13.4$ $\mathrm{mm} \mathrm{Hg})$ decreased from that of $\mathrm{BBP}(108.5 \pm 17.9 \mathrm{~mm} \mathrm{Hg})$. After $\mathrm{PIH}$ treatment, mean systolic BP1 $(166.4 \pm 19.3 \mathrm{~mm} \mathrm{Hg})$ was elevated by $24.8 \% \pm 19.3 \%$ from BPO $(135.3 \pm 18.6 \mathrm{~mm} \mathrm{Hg})$, and diastolic BP1 $(83.6 \pm 13.7 \mathrm{~mm} \mathrm{Hg})$ was elevated by $9.3 \% \pm 9.2 \%$ from BPO $(77.7 \pm 12.9 \mathrm{~mm} \mathrm{Hg})$. The mean PIH duration was $5.8 \pm 2.7$ days. The mean time to achievement of target BP was $7.6 \pm 4.6$ hours.

Nine patients developed hemorrhagic transformation-three in the PIH group and six in the CT2 group-and there were no 
Table 1. Comparison between the PIH and CT2 groups

\begin{tabular}{|c|c|c|c|}
\hline Variable & $\mathrm{PIH}(n=121)$ & CT2 $(n=121)$ & $P$ \\
\hline Intravenous tPA* & $5(4.1)$ & $7(5.8)$ & 0.769 \\
\hline Intra-arterial endovascular therapy & $11(9.1)$ & $18(14.9)$ & 0.166 \\
\hline Initial glucose level $(\mathrm{mg} / \mathrm{dL})^{+}$ & $129(109-167)$ & $130(106-184.5)$ & 0.862 \\
\hline Initial systolic BP (mm Hg) & $152.3 \pm 25.4$ & $144.6 \pm 21.5$ & 0.011 \\
\hline Initial diastolic BP ( $\mathrm{mm} \mathrm{Hg})$ & $86.6 \pm 16.4$ & $80.9 \pm 12.0$ & 0.086 \\
\hline Early neurological deterioration & $104(86)$ & $23(19)$ & 0.000 \\
\hline Initial NIHSS score ${ }^{\dagger}$ & $5(3-7)$ & $3(1-6)$ & 0.000 \\
\hline Highest NIHSS score during admission ${ }^{+}$ & $8(6-11.5)$ & $3(2-6)$ & 0.000 \\
\hline Discharge NIHSS score ${ }^{+}$ & $5(2-7.5)$ & $2(0.5-4.5)$ & 0.000 \\
\hline Delta NIHSS score ${ }^{+}$ & $4(2-5.5)$ & $1(0-3)$ & 0.000 \\
\hline Favorable $\mathrm{mRS}$ at discharge & $60(49.6)$ & $96(79.3)$ & 0.000 \\
\hline Favorable $\mathrm{mRS}$ at 3 months & $84(73.7)$ & $99(84.6)$ & 0.059 \\
\hline
\end{tabular}

Values are presented as number (\%), median (interquartile range), or mean \pm standard deviation.

$\mathrm{PIH}$, pharmacologically induced hypertension; $\mathrm{CT} 2$, patients with an additional stroke subtype; tPA, tissue plasminogen activator; BP, blood pressure; NIHSS, National Institutes of Health Stroke Scale; mRS, modified Rankin Scale.

Discrete data suing the chi-square test or *Fisher's exact test; Continuous data were analyzed using Student's t-test or ${ }^{+}$Mann-Whitney U test.

symptomatic intracerebral hemorrhages. In the CT2 group, two patients died from cerebral herniation. Among PIH patients, five experienced sinus bradycardia and one a sinus pause, which eventually improved. One patient developed vasogenic edema, one experienced renal failure, and one pulmonary hypertension. Among the CT2 patients, one developed sinus pause, one renal failure, and one pulmonary hypertension.

The aggravation of neurological deficit (highest NIHSS score-initial NIHSS score) was greater in PIH patients than in CT2 patients (3.7 vs. 1.0, $P<0.001$ ). PIH patients less often experienced favorable outcomes than CT2 patients at discharge but exhibited greater resolution of neurological deficit (delta NIHSS [highest NIHSS score-discharge NIHSS score]) at discharge. Of 110 patients in whom mRS was assessed at 3 months, the difference in the proportion of favorable $m R S$ score was marginal ( $P=0.059$ ) (Table 1). Fifty-four PIH patients (44.6\%) exhibited "early-response," in whom NIHSS score was significantly lower after 4 days than in those without early response (Figure 1). Their functional status was significantly better at discharge and at 3 months (Table 2), although the difference was not apparent when $\mathrm{mRS} \leq 2$ was used as a "favorable" outcome ( $P=0.276$ and $P=0.156$, respectively).

$\mathrm{PIH}$ was more often performed in patients with SVD and LAD than in those with cardioembolism, which is in agreement with the observation that the pathogenesis of END in patients with LAD or SVD is associated with impaired perfusion., ${ }^{9,10}$ Although END may also result from other mechanisms, such as edema development or hemorrhagic transformation, ${ }_{1}^{8} \mathrm{PIH}$ did not increase the incidence of adverse effects in our patients. Thus,

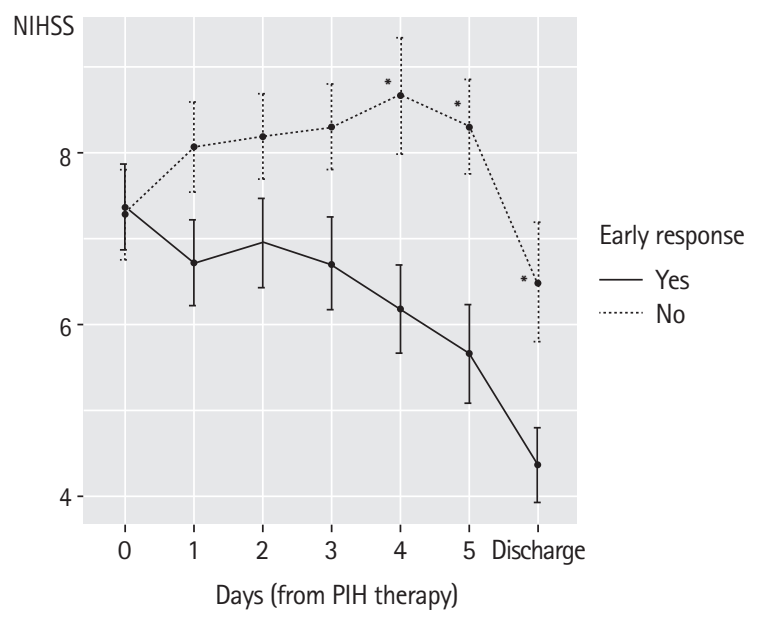

Figure 1. Daily changes in National Institutes of Health Stroke Scale (NIHSS) score (mean) from the day of initiation of pharmacologically induced hypertension (PIH) therapy according to early responsiveness. ${ }^{*} P<0.05$.

$\mathrm{PIH}$ is feasible and safe, if performed carefully.

As this was not a randomized controlled study, we were unable to evaluate the efficacy of PIH therapy. Initial neurological deficits were more severe in those with PIH than in CT2 patients, and most patients in the PIH group experienced END. However, resolution of neurological deficits (delta NIHSS) was more marked in the PIH group, and mRS scores at 3 months post-stroke did not differ between the two groups, suggesting the potential benefit of PIH therapy. Among patients receiving $\mathrm{PIH}$, the final functional outcome was significantly better in early responders than in the others (Table 2 and Figure 1), suggesting that early response may predict a better final function- 
Table 2. Neurological outcomes according to early responsiveness to pharmacologically induced hypertension

\begin{tabular}{lccc}
\hline Variable & Early response $(n=54)$ & No early response $(\mathrm{n}=67)$ & $P$ \\
\hline Initial NIHSS score & $5(2-7)$ & $5(3-8)$ & 0.295 \\
Highest NIHSS score & $7(6-11)$ & $9(7-12)$ & 0.122 \\
NIHSS score at discharge & $4(2-6)$ & $6(3-8)$ & 0.013 \\
Delta NIHSS score & $4(2-6)$ & $3(1-5)$ & 0.287 \\
Favorable mRS at discharge & $34(63.0)$ & $26(38.8)$ & 0.014 \\
Favorable mRS at 3 months & $43(86.0)$ & $41(64.1)$ & 0.015
\end{tabular}

Values are presented as median (interquartile range) or number (\%). Continuous data were analyzed using the Mann-Whitney U test and discrete data using the chi-square test.

NIHSS, National Institutes of Health Stroke Scale; mRS, modified Rankin Scale.

al outcome. Although this was an uncontrolled, observational study involving a relatively small number of patients, our data suggest that PIH therapy in patients with END is feasible, safe, and potentially effective, warranting further controlled trials to confirm its efficacy.

\section{References}

1. Oh MS, Yu KH, Hong KS, Kang DW, Park JM, Bae HJ, et al. Modest blood pressure reduction with valsartan in acute ischemic stroke: a prospective, randomized, open-label, blinded-end-point trial. Int J Stroke 2015;10:745-751.

2. Park JH, Ovbiagele B, Hong KS, Kwon SU. Association of systolic blood pressure with progression of symptomatic intracranial atherosclerotic stenosis. J Stroke 2017;19:304-311.

3. Rordorf G, Koroshetz WJ, Ezzeddine MA, Segal AZ, Buonanno FS. A pilot study of drug-induced hypertension for treatment of acute stroke. Neurology 2001;56:1210-1213.

4. Hillis $A E$, Ulatowski JA, Barker PB, Torbey M, Ziai W, Beauchamp NJ, et al. A pilot randomized trial of induced blood pressure elevation: effects on function and focal perfusion in acute and subacute stroke. Cerebrovasc Dis 2003;16:236-246.

5. Marzan AS, Hungerbühler $\mathrm{HJ}$, Studer $A$, Baumgartner RW Georgiadis D. Feasibility and safety of norepinephrine-induced arterial hypertension in acute ischemic stroke. Neurology 2004;62:1193-1195.

6. Bogoslovsky T, Häppölä O, Salonen O, Lindsberg PJ. Induced hypertension for the treatment of acute mca occlusion beyond the thrombolysis window: case report. BMC Neurol 2006;6:46.
7. Koenig MA, Geocadin RG, de Grouchy M, Glasgow J, Vimal S, Restrepo $\mathrm{L}$, et al. Safety of induced hypertension therapy in patients with acute ischemic stroke. Neurocrit Care 2006;4: 3-7.

8. Seners P, Turc G, Oppenheim C2, Baron JC. Incidence, causes and predictors of neurological deterioration occurring within $24 \mathrm{~h}$ following acute ischaemic stroke: a systematic review with pathophysiological implications. J Neurol Neurosurg Psychiatry 2015;86:87-94.

9. Alawneh JA, Moustafa RR, Baron JC. Hemodynamic factors and perfusion abnormalities in early neurological deterioration. Stroke 2009;40:e443-e450.

10. Nah HW. Small vessel transient ischemic attack and lacunar infarction detected with perfusion-weighted MRI. J Stroke 2017;19:365-366.

Correspondence: Jong S. Kim

Department of Neurology, Asan Medical Center, University of Ulsan College of Medicine, 88 Olympic-ro 43-gil, Songpa-gu, Seoul 05505, Korea

Tel: $+82-2-3010-3442$

Fax: +82-2-474-4691

E-mail: jongskim@amc.seoul.kr

Received: February 25, 2019

Revised: May 9, 2019

Accepted: May 9, 2019

This work was supported by a grant from the Ministry for Health, Welfare and Family Affairs, Republic of Korea (HI14C1985).

The authors have no financial conflicts of interest. 